\title{
Analytical calculation of the deflection of the lattice truss
}

\author{
Mikhail Kirsanov ${ }^{1, *}$, and Dmitriy Tinkov ${ }^{1}$ \\ ${ }^{1}$ Moscow State University of Civil Engineering, Yaroslavskoe shosse, 26, Moscow, 129337, Russia
}

\begin{abstract}
An algorithm is given for deriving the dependence of the deflection of a planar statically determinate beam truss on the number of panels, dimensions and load. Three load cases are considered: uniform load on the lower belt, upper belt and vertical force in the middle of the span. By induction, generalizing a series of solutions for trusses with a consecutively increasing number of panels, the desired formula is obtained for the deflection and horizontal displacement of the mobile support of the truss. All transformations are performed in the system of symbolic mathematics Maple. For a sequence of coefficients of the desired formula, using the special Maple operators, homogeneous recurrent equations are constructed and solved. The coefficients found are in the form of polynomials in the number of panels. The asymptotic property of the solution is found. On the graphs of the dependence of the deflection on the number of panels and on the height, extreme points are found. The solution can be used to test the calculations obtained numerically.
\end{abstract}

\section{Introduction}

In design practice, all calculations for the strength, stability and endurance of rod structures are performed numerically [1-5]. Optimization problems are also solved numerically [6-9]. Analytical calculations of building structures are quite rare, and the formulas on which they are based generally have a narrow field of application. The calculation of a particular truss with dimensions and loads, designated as variable parameters, is quite simple. For this purpose, known methods of determining the forces in rods and the displacement of nodes are applied in any program of symbolic mathematics (Maple, Mathematica, Derive, Reduce, etc.). Without special difficulties it is possible to obtain a formula for determining the desired quantity and changing the parameters included in the formula to choose the most profitable project. It is much more difficult to obtain the dependence of forces or displacements on the number of panels or rods of a truss if a calculated design has a periodic structure with a certain type of periodicity cell. As a periodicity cell, you can consider as one truss panel or several. For such a truss that in the present paper the task is to obtain a formula for the dependence of the deflection of a truss on the number of panels. A method is used to induct generalizations of a number of solutions for trusses with a consecutively increasing number of panels. Previously, this method was

\footnotetext{
* Corresponding author: c216@ya.ru
} 
used in [10-13] to solve similar problems for planar and spatial [14-16] trusses. In [17] by induction method, a solution was obtained for the deformation of the pile foundation. The inductive method in the problem of the deflection of a truss taking account of the creep of the material was solved in [18].

\section{Truss and Methods}

A planar truss (Fig. 1) with a height of $4 h$ and a span length of $L=a(6 n+2)$ in which $n$ periodicity cells are separated has two supports and $m=24 n+12$ rods, including three support rods (one in the left support, two in the right). The lattice of the truss consists of $18 n$ +3 racks, of which six lateral are shortened, and $6 n$ braces are $d=\sqrt{9 a^{2}+16 h^{2}}$ in length. Two angular lateral braces are long of $c=\sqrt{a^{2}+h^{2}}$.

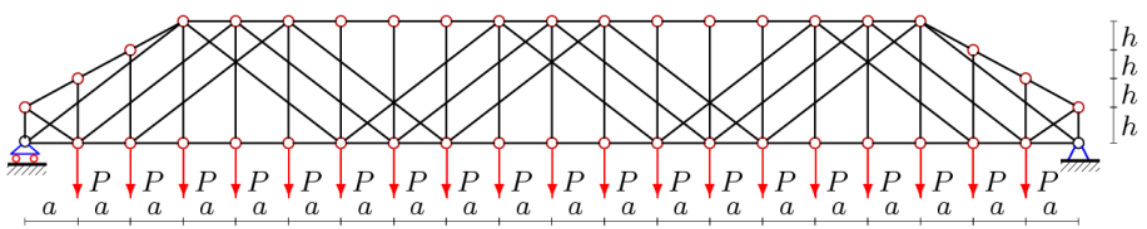

Fig. 1. Truss, load on the lower belt $n=3$

Calculation of the forces in elements required to calculate the deflection in a symbolic form is performed by cutting out the nodes in the computer mathematics system Maple. The matrix of the system of equilibrium equations of nodes is filled in a cycle according to the number of rods. Elements of the matrix are the direction cosines of unknown forces in the rods. They are determined from the coordinates of the ends of the rods. Hinges and rods are numbered (Figure 2). The origin is in the left reference node of the truss.

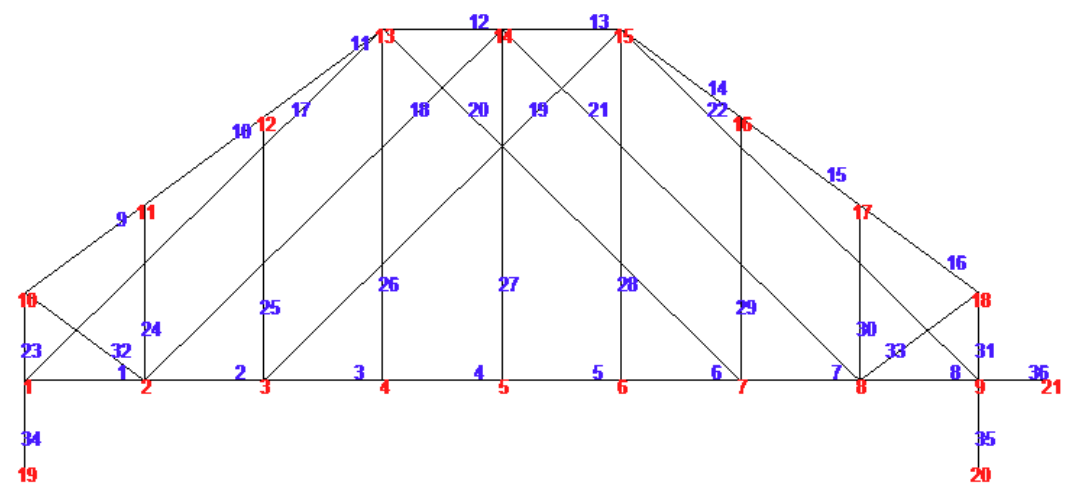

Fig. 2. Numbering of hinges and rods in the Maple system, $n=1$

The fragment of the program for entering coordinates of the nodes of the lower belt in the language of symbolic mathematics Maple has the form

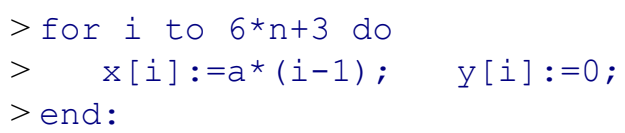


To enter the connection structure into the program, vectors $\mathrm{N}$ [ i ] are used that coincide with the rods of the truss with some conditional direction. The coordinates of these vectors are the numbers of the ends of the rod. For example, you enter, for example, a truss belt

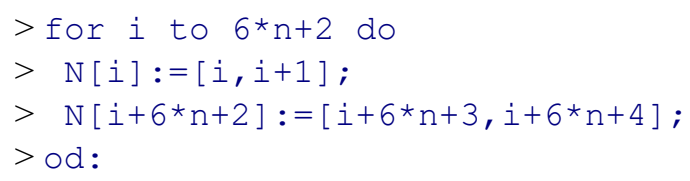

After this, the matrix $\mathrm{G}$ of the system of equations of node equilibrium is formed. In the odd-numbered lines, cosines with the horizontal $x$-axis are introduced, in even-numbered cases - with a vertical $y$ :

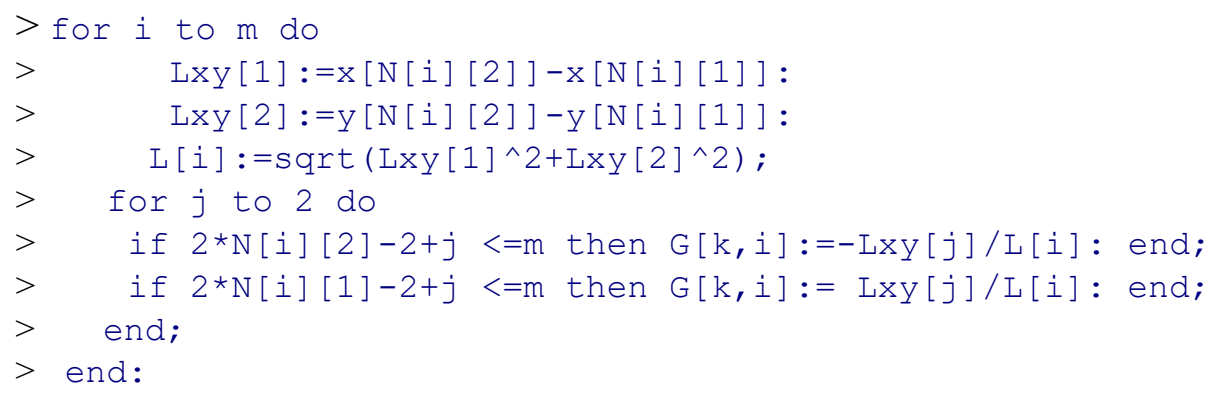

Here is denoted: Lxy — projections of conditional vectors - rods on the coordinate axis. From the solution of the matrix equation $G \bar{S}=\bar{B}$, where $\bar{S}-$ is the vector of all forces in the rods, $\bar{B}$ - the load vector, the forces are obtained in the symbolic form. The load vector, as well as the matrix, consists of horizontal forces in odd elements and vertical forces in even ones. The displacement of the middle node of the lower belt is determined by the MaxwellMohr's formula

$$
\Delta=\sum_{i=1}^{m-3} S_{i}^{(P)} S_{i}^{(1)} l_{i} /(E F)
$$

It is indicated: $S_{i}^{(P)}$ - the forces in the rods from the given load, $l_{i}$ - the length of the rods, $S_{i}^{(1)}$ - the forces from the unit force applied to the knot of the lower belt in the middle of the span, $E F$ - the rigidity of the rods.

Consider first the case of loading the lower belt (Figure 1). The vector of the right-hand side is organized as follows

>for $i$ from 2 to $6 *^{*}+2$ do $B[2 * i]:=1$ : end:

\section{Results}

The solution for trusses with an arbitrary number of panels has the form

$$
\Delta=P\left(C_{1} a^{3}+C_{2} h^{3}+C_{3} c^{3}+C_{4} d^{3}\right) /\left(16 h^{2} E F\right) .
$$


The calculation of a series of trusses reveals sequences of coefficients for $a^{3}, h^{3}, c^{3}$ and $d^{3}$. Operators of the Maple system rgf_findrecur and rsolve from the solution of recurrence equations give the following patterns

$$
\begin{aligned}
& C_{1}=\left(135 n^{4}+180 n^{3}+81 n^{2}-50 n+14\right) / 4, \\
& C_{2}=32\left(2 n+2-(-1)^{n}\right), \\
& C_{3}=32(2 n+1), \\
& C_{4}=(n-1) / 2 .
\end{aligned}
$$

For the coefficient $C_{1}$, a recurrence equation of the fifth order $C_{1, n}=5 C_{1, n-1}-10 C_{1, n-2}+10 C_{1, n-3}-5 C_{1, n-4}+C_{1, n-5}$ was obtained and solved, and for the coefficient $C_{2}$ the equation $C_{2, n}=C_{2, n-1}+C_{2, n-2}-C_{2, n-3}$.

Expression (1) with coefficients (2) gives the solution of the problem posed for the load along the lower belt of the truss.

Similarly, for the load on the upper belt of the truss (Figure 3), we obtain the deflection in the form (1) and the same coefficients (2), except for the coefficient at $h^{3}$, which takes the form $C_{2}=65(n+1)$. The vector of the right side in this case is given as follows

$>$ for $i$ from $6 * n+4$ to $12 * n+6$ do $B p[2 * i]:=1$ : end:

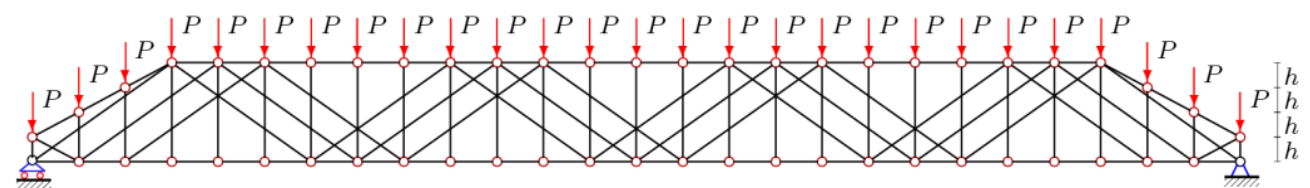

$a, a, a, a, a, a, a, a, a, a, a, a, a, a, a, a, a, a, a, a, a, a, a, a, a, a$

Fig. 3. Truss, load on the upper belt $n=4$

The simpler coefficients in (1) will be in calculating the deflection from one force in the middle of the lower belt (Figure 4):

$$
\begin{aligned}
& C_{1}=\left(18 n^{3}+18 n^{2}+15 n+7\right) / 2, \\
& C_{2}=32\left(2-(-1)^{n}\right), \\
& C_{3}=32, \\
& C_{4}=(n+1) / 2 .
\end{aligned}
$$

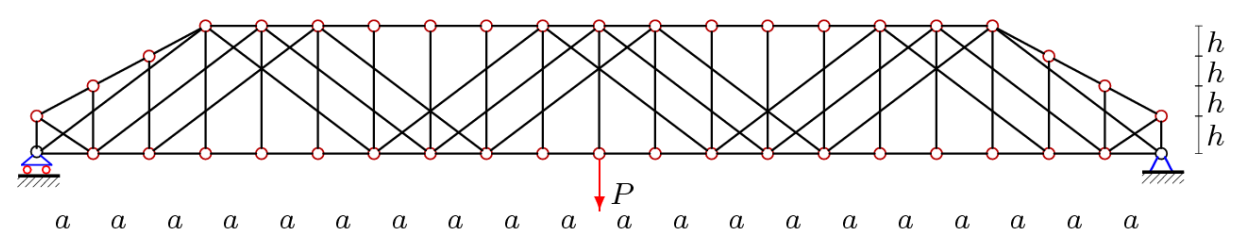

Fig. 4. Truss, load on the center, $n=3$ 


\section{Discussion}

The main advantage of the solution (1) is the dependence of the deflection on the number of panels. This allows, first, to apply formula (1) for a wide class of problems, and secondly, to find some qualitative effects of such a solution, which is somewhat difficult in the framework of numerical calculations. We give some examples. We introduce the dimensionless deflection $\Delta^{\prime}=\Delta E F /\left(P_{0} L\right)$. Figure 5 shows the curves of the obtained dependence at $L=100 m, P_{0}=P(6 n+3)$. Horizontal asymptotes are obvious

$$
\Delta_{\max }=\lim _{n \rightarrow \infty} \Delta^{\prime}=\frac{5 L^{3}+5120 h^{3}}{3072 h^{2} L} .
$$

One of these straight lines is indicated in Fig. 5 for $h=2.00 \mathrm{~m}$. The curves also have an extremum point. The number of panels at which the minimum is reached is almost independent of the height.

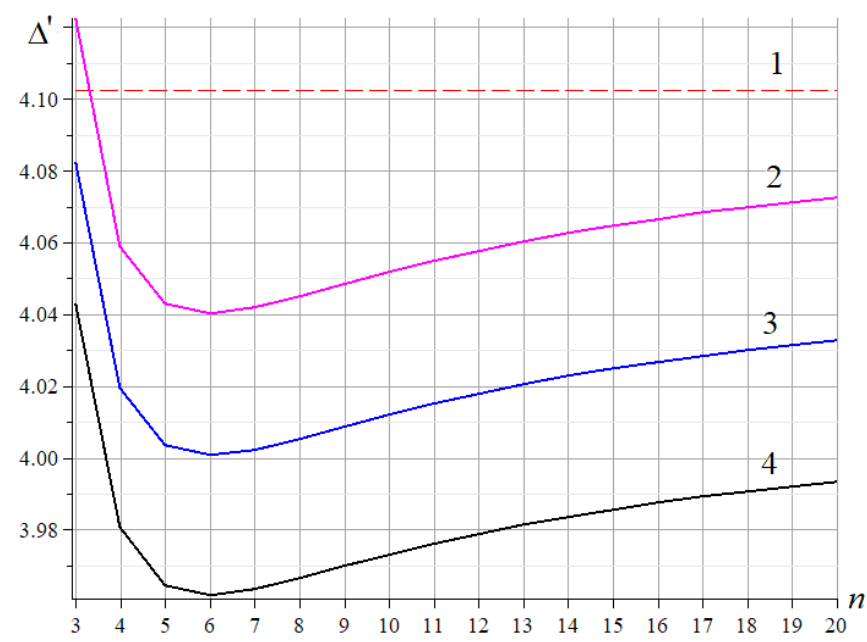

Fig 5. Dependence of the dimensionless deflection on the number of panels during loading on the upper belt. $1-4_{\max } ; 2-h=2.00 \mathrm{~m}, 3-h=2.01 \mathrm{~m}, 4-h=2.02 \mathrm{~m}$

There is one more feature in the solution. If you plot the curves of the deflection versus the height of the truss, you can also notice extreme points (Figure 6). Calculations are performed for the span $L=30 \mathrm{~m}$. With an increase in the number of panels for this size of the span, the deflection decreases. For large spans $L>80 \mathrm{~m}$, similar curves no longer have minimum points. The deflection decreases monotonically with increasing altitude and begins to grow starting from unrealistically large heights of the structure. 


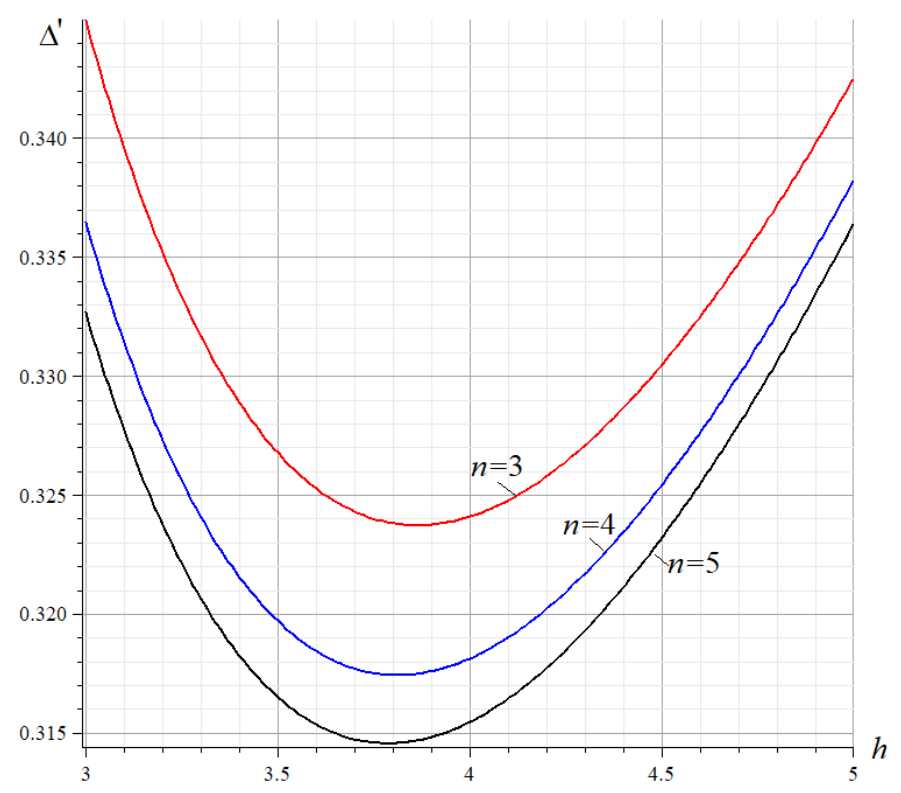

Fig 6. Dependence of the dimensionless deflection on the height of the truss under load on the upper belt

In addition to the size of the vertical deflection, the deformability of the truss can be estimated from the horizontal displacement of the mobile support from the action of the loads. The horizontal displacement of the support is determined by the Maxwell-Mora formula

$$
\Delta=P \sum_{i=1}^{m-3} S_{i}^{(P)} S_{i}^{(2)} l_{i} /(E F) .
$$

where $S_{i}^{(2)}$ — the forces from the unit horizontal force applied to the left support. Omitting the intermediate calculations, we give the formula for the displacement of the support from the action of loads along the lower belt (Figure 1)

$$
\delta_{A}=3 P a^{2}\left(6 n^{3}+6 n^{2}+4 n-1\right) /(4 h E F) .
$$

When the upper belt is loaded, the displacement is the same, and when the concentrated load acts in the middle of the span, the displacement has the form

$$
\delta_{A}=3 P a^{2}\left(6 n^{2}-3(-1)^{n}+4 n-1\right) /(16 h E F) .
$$

\section{Conclusions}

The new sheme of considered truss has a rather complex structure, while remaining statically determinate. Despite this, an accurate analytical dependence on the number of panels has been obtained for the bending of the truss. Calculations of individual examples showed the presence of extreme points in the dependence found, which makes possible some optimization of the rigidity design. There are known works on optimization of trusses for rigidity, economy, strength and stability [6-9]. The obtained analytical formulas with sufficiently simple polynomial coefficients can supplement these results or evaluate their 
accuracy and reliability. It is quite obvious that the solutions found in this paper can be extended to statically indeterminate constructions. In this case, the above formulas are the solution of the basic problem of the method of forces. A survey of papers on the application of the induction method in problems on planar trusses was made in [19].

\section{References}

1. K.A. Egarmin, G.Yu. Sysoev, N.I. Vatin, M.V. Vrublevskaya, Construction of Unique Buildings and Structures 2, 34 (2015)

2. M. Y. Baranovskiy, V. A. Tarasov, Construction of Unique Buildings and Structures 7, 92 (2014)

3. A.V. Alekseytsev, N.S. Kurchenko, Magazine of Civil Engineering, 5, 3 (2017)

4. I.N. Serpik, A.V. Alekseytsev, P.Yu. Balabin, N.S. Kurchenk, Magazine of Civil Engineering, 8, 181 (2017)

5. T. Saknite, D. Serdjuks, V. Goremikins, L. Pakrastins, N.I. Vatin, Magazine of Civil Engineering, 4, 26 (2016)

6. A. Kaveh, V. R. Mahdavi, Structural Engineering and Mechanics 5, 847 (2015)

7. A. Kaveh, A. Zolghadr, Applied Soft Computing Journal, 5, 2727 (2013)

8. A. Ahrari, D. Kalyanmoy, Computers \& Structures 164, 127 (2016)

9. I.N. Serpik, A.V. Alekseytsev, Magazine of Civil Engineering, 1, 14 (2016)

10. M.N. Kirsanov, N. Zaborskaya, Magazine of Civil Engineering, 3, 61 (2017)

11. M.N. Kirsanov, Magazine of Civil Engineering, 1, 33 (2016)

12. M.N. Kirsanov, Magazine of Civil Engineering, 5, 58 (2015)

13. M.N. Kirsanov, Magazine of Civil Engineering, 4, 38 (2015)

14. M.N. Kirsanov, Magazine of Civil Engineering, 4, 52 (2016)

15. M.N. Kirsanov, Magazine of Civil Engineering, 8, 58 (2017)

16. M.N. Kirsanov, Magazine of Civil Engineering, 1, 32 (2015)

17. D.V. Tinkov, Magazine of Civil Engineering, 1, 25 (2016)

18. M.N. Kirsanov, Magazine of Civil Engineering, 3, 3 (2015)

19. D.V. Tinkov, Magazine of Civil Engineering, 5, 66 (2015) 